\title{
DEVELOPMENT OF A MONOTONIC MULTIDIMENSIONAL ADVECTION-DIFFUSION SCHEME
}

\author{
S. K. Wang, Tony W. H. Sheu, and S. F. Tsai \\ Department of Naval Architecture and Ocean Engineering, National Taiwan \\ University, 73 Chou-Shan Rd., Taipei, Taiwan, Republic of China
}

\begin{abstract}
The objective of this study is to present a finite-element ad vection-diffusion scheme for the steady scalar transport equation. The novelty is the use of two ad vection-diffusion schemes in combination in a way which ensures the satisfaction of the monotonicity property in their matrix equation. Common to these two fundamental finite-element models is that matrix equations are all classified to be irreducibly diagonal dominant. The resulting M-matrix finite-element method is the method of choice to resolve sharp profiles in the flow. The first finite-element method unconditionally provides monotonic solutions. The gain in the stability is due to the introduction of the upwind information along the local streamline. The second basic scheme is classified as conditionall y monotonic and is well suited to predicting lower Peclet number flows. This Petrov-Galerkin finite-element model manifests itself by the use of Legendre polynomials to span finite-element spaces. An inherent feature of this formulation is the orthogonal property, which enables a considerable saving in the numerical evaluation of integral terms. Computational evidence reveals that the Legendrepolynomial finite-element model can provide more accurate solutions in low Peclet number conditions. As the Peclet number is increased to higher values that forbid a monotonic solution, the unconditionally monotonic finite-element model is used to complement the Legendre-polynomial finite-element model. This helps enhance the stability. A combined formulation renders a composite scheme that offers promise to optimize the scheme performance. In order to show that the present composite scheme is computationally efficient, the method needs to be rigorously tested against a vailable analytic results. This composite scheme was found to provide monotonic solutions under high and low Peclet number conditions and provided accurate solutions at less computational cost. Use of this composite scheme promises a wider range of practical problems that can be modeled numerically.
\end{abstract}

\section{INTRODUCTION}

In the physical sciences and engineering, Navier-Stokes flows may be far too complex and an in-depth assessment of discretization schemes is seldom possible. Therefore, it is preferred to consider the scalar convection-diffusion equation, which is the simplest prototype equation capable of characterizing these flows. This

\section{Received 6 January 1999; accepted 25 June 1999}

The authors wish to express their sincere gratitude to the Computer Center of National Taiwan University and the National Center for High-Performance Computing (NCHC) for supporting this study through the use of Cray J916 and IBM RS/6000-590 computers, which made this study possible. Financial support by the National Science Council under grant NSC 87-2213-E-002-00 2 is also gratefully acknowledged.

Address correspondence to Prof. Tony W. H. Sheu, Institute of Naval Architecture, National Taiwan University, 73 Chou-Shan Rd., Taipei, Taiwan, Republic of China. E-mail: sheu@indy.na.ntu.edu.tw 


\begin{tabular}{|c|c|c|c|}
\hline \multicolumn{4}{|c|}{ NOMENCLATURE } \\
\hline$h$ & mesh size & $W_{i}$ & weighting function \\
\hline$h_{\xi}, h_{\eta}$ & mesh sizes in the computational plane & & optimum factor \\
\hline & Jacobian of the Transformation & & permutation notation \\
\hline$N_{i}$ & basis function & $\Delta x, \delta y$ & mesh size along the $x$ and $y$ \\
\hline $\mathrm{Pe}$ & Peclet number & & directions, respectively \\
\hline$P_{i}$ & $i$ th term of the Legendre polynomial & $\mu$ & diffusivity of the fluid \\
\hline$s$ & streamline coordinate & $\xi, \eta$ & normalized spatial coordinates \\
\hline$U, V$ & $\begin{array}{l}\text { ratio of ve locity components } u, v \text { to } \\
\text { diffusivity } \mu\end{array}$ & $\Phi$ & $\begin{array}{l}\text { primary variable for the scalar transport } \\
\text { equation }\end{array}$ \\
\hline$u, v$ & $\begin{array}{l}\text { velocity component in } x \text { and } y \\
\text { directions, respectively }\end{array}$ & & \\
\hline
\end{tabular}

model equation is also a very useful vehicle for benchmarking the discretization methods so far devised, since it is more amenable to analytic solutions. This explains in part why this model problem is still the subject of much research interest, academically as well as practically.

Over the years, a great deal of research effort has been dedicated to resolving difficulties incurred by the prevailing convection terms. A more fundamentally challenging problem encountered in science and industry is due to wiggles which steepen ahead of discontinuities, further complicating the analysis. The need to wipe out these oscillatory solutions was the main motivation for the present study. To develop a discretization scheme which is computationally efficient, it is also crucial to take solution accuracy, computational efficiency, and ease of programming into account concurrently. Retaining these computationally desirable properties is a difficult task. What fits for solution accuracy may often not fit for solution stability. In this study, solution monotonicity and solution accuracy serve as two design guidelines behind our construction of the advection-diffusion scheme.

The key to conducting an accurate multidimensional flow simulation is to prevent cross-wind diffusion (or false diffusion) errors. It is most effective to circumvent this difficulty by introducing flow-oriented mechanism into the formulation. As alluded to earlier, steep gradients appear which can cause the solution to deteriorate further. Under these circumstances, a flow-oriented flux discretization scheme no longer suffices for production of oscillation-free solutions. In attempting to suppress over- or under-shoots in the solution, researchers have developed bounding schemes. Many of these schemes have been developed to accommodate the total variation diminishing (TVD) property [1]. However, extension of TVD schemes to multidimensional flow simulations has not yet been placed on a rigorous analytic foundation.

In the development of oscillation-free multidimensional schemes, one has the choice to apply a global positivity principle $[2,3]$ to explicit schemes. While this principle has a sound theoretical basis and is easy to implement in existing computer codes, the limited application scope forbids the use of this idea to analyses which involve solving field variables from a simultaneous set of algebraic equations. This has prompted the current research into an alternative filtering technique which is applicable to finite-element analyses. Inspired by the work of 
Ahue and Telias [4], we constructed a weighting function which favors the field variable at the upstream side [5-8]. Guided by the theoretically rigorous theory of the M-matrix $[9,10]$, an attempt has been made to construct weighting functions in order to guarante e monotonic solutions even in the flow having sharp gradients or discontinuities.

The organization of this article is as follows. We begin by describing the convection-diffusion model equation. This is followed by an introduction of two underlying monotonicity-preserving finite-element models. We have employed these monotonic schemes in combination to obtain higher solution accuracy without compromising stability. For validation purposes, we will consider two problems which have closed-form solutions in a square cavity. Two numerical examples are also considered, both of which have high-gradient solutions. A key issue that underlies the present work is to provide a means of determining the weighting factor to optimize the composite scheme. Finally, some conclusions are drawn.

\section{MULTIDIMENSIONAL FINITE-ELEMENT MODELS}

The working equation is that of the steady-state equation for simulating the scalar $\Phi$ transported in the two-dimensional flow:

$$
U \Phi_{x}+V \Phi_{y}=\left(\Phi_{x x}+\Phi_{y y}\right)
$$

In the above equation, $U$ and $V$ are defined by $U=u / \mu$ and $V=v / \mu$. For ease of illustration, we will restrict our attention to a simple flow. In all cases investigated, the flow considered is with constant velocity components $u$ and $v$. Another assumption made here is that diffusivity of the fluid, $\mu$, is kept constant throughout the analysis. When solving the elliptic partial differential equation, we demand specification of boundary data of $\Phi$ on the entire boundary of the simply connected domain $D$.

Our strategy for obtaining finite-element solutions, $\hat{\Phi}$, to the model equation (1) is to make the residual $R=U \hat{\Phi}_{x}+V \hat{\Phi}_{y}-\left(\hat{\Phi}_{x x}+\hat{\Phi}_{y y}\right)$ orthogonal to the weighting function. The solutions thus computed can be viewed as a search for the weak solutions to Eq. (1). In the weak formulation, care must be taken to devise weighting (or test) functions appropriately as the maximum values of $\mathrm{Pe}_{x}=U \Delta x$ and $\mathrm{Pe}_{y}=V \Delta y$ greatly exceed the critical value of 2 . The analysis is followed by a substitution of bilinear basis functions, say $N_{i}$ for $\hat{\Phi}=\Sigma_{i=1}^{4} N_{i}(\xi, \eta) \Phi_{i}$, into the weighted residual statement to obtain stiffness matrices for all elements. The analysis is followed by a finite-element assemblage to form a global coefficient matrix.

In attempting to enhance the finite-element equation, we assign spatially unequal weights to convective terms so that field variables at the upwind side are favored. It has to be remarked that diffusive fluxes need to be unequally weighted to render a consistent weighted-residual finite-element model [11]. For this study, the chosen weighting functions are as follows [7]:

$$
W_{i}=D_{i}\left[d_{\xi 0} P_{0}(\xi)+d_{\xi 1} P_{1}(\xi)\right]\left[d_{\eta 0} P_{0}(\eta)+d_{\eta 1} P_{1}(\eta)\right]
$$


In a discretized domain with grid spacings $h_{\xi}$ and $h_{\eta}$, the coefficients shown in Eq.

(2) are as follows:

$$
D_{i}=\frac{1}{4} \exp \left(\frac{u h_{\xi} \xi_{i}}{2 \mu}\right) \exp \left(\frac{v h_{\eta} \eta_{i}}{2 \mu}\right)
$$

where

$$
\begin{aligned}
d_{\xi 0} & =\frac{1}{2} \int_{-1}^{1} W_{\xi}(t) P_{0}(t) d t \\
d_{\xi 1} & =\frac{3}{2} \int_{-1}^{1} W_{\xi}(t) P_{1}(t) d t \\
d_{\eta 0} & =\frac{1}{2} \int_{-1}^{1} W_{\eta}(t) P_{0}(t) d t \\
d_{\eta 1} & =\frac{3}{2} \int_{-1}^{1} W_{\eta}(t) P_{1}(t) d t \\
W_{\xi}(\xi) & =\left(1+\xi_{i} \xi\right) \exp \left(-\frac{u h_{\xi} \xi}{2 \mu}\right) \\
W_{\eta}(\eta) & =\left(1+\eta_{i} \eta\right) \exp \left(-\frac{v h_{\eta} \eta}{2 \mu}\right)
\end{aligned}
$$

In Eq. (2), we have tacitly used Legendre polynomials $P_{0}(t)=1$ and $P_{1}(t)=t$ to span finite-element spaces. The advantage of choosing Legendre polynomials becomes apparent in view of the following orthogonal property:

$$
\int_{-1}^{+1} P_{i}(t) P_{j}(t) d t=\frac{2}{2 i+1} \delta_{i j} \quad(i \text { is a dummy index })
$$

It was in recognition of this fact that we rewrite the bilinear shape functions $N_{i}(\xi, \eta)$ in terms of Legendre polynomials $P_{0}$ and $P_{1}$ as follows:

$$
N_{i}(\xi, \eta)=\frac{1}{4}\left[P_{0}(\xi)+\xi_{i} P_{1}(\xi)\right]\left[P_{0}(\eta)+\eta_{i} P_{1}(\eta)\right]
$$

The use of Legendre polynomials to span finite-element spaces results in an substantial reduction of the computational effort in calculating integrals. The tremendous gain in computation speed and save in storage greatly expands the application scope.

It has been known for quite some time that a real, irreducible, diagonally dominant matrix is the key to obtaining a monotonic solution profile. One major characteristic of this matrix is its off-diagonal entries, which are nonpositive. Guided by the maximum principle [4], the present study was undertaken to check 
whether the analysis formulated within the Legendre-polynomial finite-element framework is classified as an unconditionally monotonic solution. To this end, we can derive a compact discrete equation of (1) which involves a nine-point computational molecule. All coefficients in the discrete equation are functions of the Peclet number. By varying the values of $\mathrm{Pe}_{x}$ and $\mathrm{Pe}_{y}$, we can determine under what conditions the sufficient (but not necessary) condition for obtaining a monotonic solution can be derived. As $\mathrm{Pe}_{x}$ and $\mathrm{Pe}_{y}$ are smaller than 3.6, the computed solutions will, by definition, be monotonic. The need to perform calculations in this fairly restricted Peclet number range places limitations on the computational techniques as a practical tool when conducting a large-scale simulation. Thus, we have a strong need to extend the monotonic stability range for improving the computational efficiency. In this study, we consider the scheme of Rice and Schnipke [3] as an aid to resolve this problem.

As Rice and Schnipke [3] did, we take $u_{s} \Phi_{s}\left(\equiv u \Phi_{x}+v \Phi_{y}\right)$ as a constant value in each element. Taking the case with $u>0$ and $v>0$ as an illustrative example, the resulting matrix equation for each element can be derived as

$$
\left(\begin{array}{cccc}
0 & 0 & 0 & 0 \\
0 & 0 & 0 & 0 \\
-F_{p} F_{n} \frac{u_{s}}{\Delta s} A_{f} & -\left(1-F_{n}\right) \frac{u_{s}}{\Delta s} A_{f} & \frac{u_{s}}{\Delta s} A_{f} & -\left(1-F_{p}\right) \frac{u_{s}}{\Delta s} A_{f} \\
0 & 0 & 0 & 0
\end{array}\right)
$$

where

$$
\begin{aligned}
A_{f} & =\iint \sum_{i=1}^{4} N_{i}(\xi, \eta)|J| d \xi d \eta \\
0 \leq F_{p} & \equiv \max \left\{\min \left(\frac{F_{1}}{F_{2}}, 1\right) 0\right\} \leq 1 \\
0 \leq F_{n} & \equiv \max \left\{\min \left(\frac{F_{4}}{F_{3}}, 1\right) 0\right\} \leq 1 \\
F_{1} & =v\left(x_{3}-x_{4}\right)+u\left(y_{4}-y_{3}\right) \\
F_{2} & =v\left(x_{1}-x_{4}\right)+u\left(y_{4}-y_{1}\right) \\
F_{3} & =v\left(x_{2}-x_{1}\right)+u\left(y_{1}-y_{2}\right) \\
F_{4} & =v\left(x_{2}-x_{3}\right)+u\left(y_{3}-y_{2}\right)
\end{aligned}
$$

Referring to Figure 1, the length of $\Delta s$ takes on the value

$$
\Delta s=\left[\left(x_{3}-x^{\prime}\right)^{2}+\left(y_{3}-y^{\prime}\right)^{2}\right]^{1 / 2}
$$




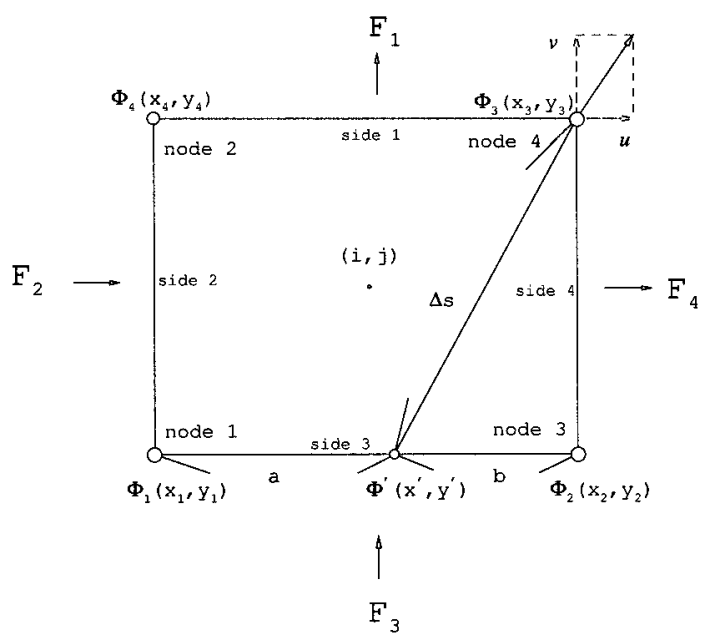

Figure 1. The notations used in the finite-element model of Rice and Schnipke.

\section{FUNDAMENTAL STUDIES ON TWO UPWIND MODELS}

With discretization schemes described above, it is then a question of checking their predictions against suitable test problems. We consider an analytic test problem to benchmark the solution accuracy and examine the scheme monotonicity of two investigated finite-element models. The model problem is schematic in Figure 2. Subject to the prescribed boundary data, the advection-diffusion equation (1) is amenable to the following boundary-layer-type solution:

$$
\Phi(x, y)=\frac{\{1-\exp [(x-1) U]\}\{1-\exp [(y-1) V]\}}{[1-\exp (-U)][1-\exp (-V)]}
$$

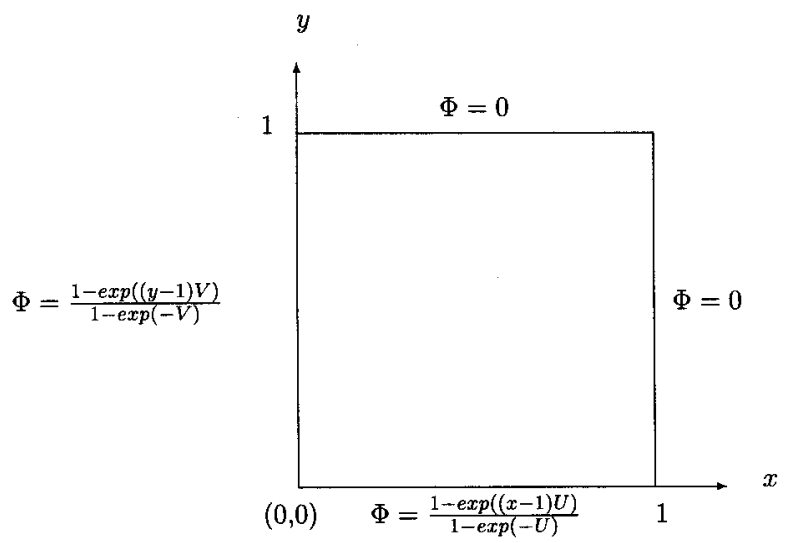

Figure 2. Schematic of the test problem for Eq. (8). 
Table 1. Computed $L_{2}$ error norms with different velocities $U, V$ and the grid numbers for Eq. (8) using the Legendre-polynomial finite-element model

\begin{tabular}{lcccccc}
\hline$U$ and $V$ & Norm & $11 \times 11$ & $21 \times 21$ & $31 \times 31$ & $41 \times 41$ & $51 \times 51$ \\
\hline$U=1$, & $L_{1}$ & $8.05 \times 10^{-8}$ & $6.26 \times 10^{-8}$ & $6.93 \times 10^{-8}$ & $5.50 \times 10^{-8}$ & $6.46 \times 10^{-8}$ \\
$V=1$ & $L_{2}$ & $1.18 \times 10^{-9}$ & $1.10 \times 10^{-9}$ & $1.12 \times 10^{-9}$ & $1.07 \times 10^{-9}$ & $1.09 \times 10^{-9}$ \\
& $L_{\infty}$ & $4.56 \times 10^{-9}$ & $4.56 \times 10^{-9}$ & $4.56 \times 10^{-9}$ & $4.56 \times 10^{-9}$ & $4.56 \times 10^{-9}$ \\
& $E_{\text {shp }}$ & $5.67 \times 10^{-8}$ & $5.22 \times 10^{-8}$ & $5.31 \times 10^{-8}$ & $4.98 \times 10^{-8}$ & $4.98 \times 10^{-8}$ \\
$U=10$, & $L_{1}$ & $8.26 \times 10^{-8}$ & $7.57 \times 10^{-8}$ & $6.93 \times 10^{-8}$ & $5.95 \times 10^{-8}$ & $5.86 \times 10^{-8}$ \\
$V=10$ & $L_{2}$ & $1.22 \times 10^{-9}$ & $1.16 \times 10^{-9}$ & $1.13 \times 10^{-9}$ & $1.11 \times 10^{-9}$ & $1.11 \times 10^{-9}$ \\
& $L_{\infty}$ & $4.26 \times 10^{-9}$ & $4.26 \times 10^{-9}$ & $4.26 \times 10^{-9}$ & $4.26 \times 10^{-9}$ & $4.26 \times 10^{-9}$ \\
& $E_{\text {shp }}$ & $7.59 \times 10^{-8}$ & $7.02 \times 10^{-8}$ & $6.52 \times 10^{-8}$ & $6.35 \times 10^{-8}$ & $6.08 \times 10^{-8}$ \\
$U=100$, & $L_{1}$ & $8.29 \times 10^{-5}$ & $1.51 \times 10^{-6}$ & $8.46 \times 10^{-9}$ & $6.17 \times 10^{-9}$ & $5.07 \times 10^{-9}$ \\
$V=100$ & $L_{2}$ & $4.41 \times 10^{-6}$ & $7.62 \times 10^{-8}$ & $3.70 \times 10^{-10}$ & $2.87 \times 10^{-10}$ & $2.67 \times 10^{-10}$ \\
& $L_{\infty}$ & $4.54 \times 10^{-5}$ & $7.70 \times 10^{-7}$ & $2.06 \times 10^{-9}$ & $2.06 \times 10^{-9}$ & $2.06 \times 10^{-9}$ \\
& $E_{\text {shp }}$ & $1.66 \times 10^{-4}$ & $2.14 \times 10^{-6}$ & $1.47 \times 10^{-8}$ & $9.77 \times 10^{-9}$ & $7.54 \times 10^{-9}$ \\
\hline
\end{tabular}

Computations were conducted on continuously refined uniform grids $11 \times 11$, $21 \times 21,31 \times 31,41 \times 41$, and $51 \times 51$. The prediction errors cast in their $L_{1}, L_{2}, L_{\infty}$, and $E_{\text {shp }}$ norms. These norms are tabulated in Tables 1-4 and are defined below:

$$
\begin{gathered}
L_{1}=\sum_{i=1}^{n}\left|\Phi_{i(\text { computed })}-\Phi_{i(\text { exact })}\right| \\
L_{2}=\left[\frac{\sum_{i=1}^{n}\left|\Phi_{i(\text { computed })}-\Phi_{i(\text { e xact })}\right|^{2}}{n}\right]^{1 / 2} \\
L_{\infty}=\max \left|\Phi_{i(\text { computed })}-\Phi_{i(\text { exact })}\right| \quad i=1, \ldots, n \\
E_{\text {shp }}=\sum_{j=1}^{m} \sum_{i=1}^{n-1}\left|\left(\Phi_{i+1, j(\text { computed })}-\Phi_{i+1, j(\text { xact })}\right)-\left(\Phi_{i, j(\text { computed })}-\Phi_{i, j(\text { xact })}\right)\right|
\end{gathered}
$$

In this study, consideration is given to the case of $1 \leq U, V \leq 10^{5}$.

As the computed errors shown in Tables 1-4, two approaches under consideration are not without complications. It is preferable to apply the Legendre-polynomial finite-element model [7] to simulate lower Peclet number flows, while for cases with higher Peclet numbers it is advantageous to apply the finite-element model of Rice and Schnipke [3]. This study reveals that oscillatory solutions are never found in this characteristic finite-element model. The Legendre-polynomial finite-element model, on the other hand, outperforms the model of Rice and Schnipke [3] in prediction accuracy. Since there is a room for improving accuracy and stability concurrently, we are motivated to apply them in combination to 
Table 2. Computed $L_{2}$ error norms with different velocities $U, V$ and the grid numbers for Eq. (8) using the finite-element model of Rice and Schnipke

\begin{tabular}{lllcccc}
\hline$U$ and $V$ & Norm & $11 \times 11$ & $21 \times 21$ & $31 \times 31$ & $41 \times 41$ & $51 \times 51$ \\
\hline$U=100$, & $L_{1}$ & 1.72 & $4.97 \times 10^{-1}$ & $2.17 \times 10^{-1}$ & $1.17 \times 10^{-1}$ & $7.16 \times 10^{-2}$ \\
$V=100$ & $L_{2}$ & $3.55 \times 10^{-2}$ & $1.09 \times 10^{-2}$ & $4.84 \times 10^{-3}$ & $2.62 \times 10^{-3}$ & $1.62 \times 10^{-3}$ \\
& $L_{\infty}$ & $1.49 \times 10^{-1}$ & $5.10 \times 10^{-2}$ & $2.34 \times 10^{-2}$ & $1.29 \times 10^{-2}$ & $8.00 \times 10^{-3}$ \\
& $E_{\text {shp }}$ & 1.73 & $5.36 \times 10^{-1}$ & $2.38 \times 10^{-1}$ & $1.29 \times 10^{-1}$ & $7.93 \times 10^{-2}$ \\
$U=1,000$, & $L_{1}$ & $1.76 \times 10^{-1}$ & $6.86 \times 10^{-3}$ & $4.43 \times 10^{-4}$ & $3.93 \times 10^{-5}$ & $4.40 \times 10^{-6}$ \\
$V=1,000$ & $L_{2}$ & $3.90 \times 10^{-3}$ & $1.54 \times 10^{-4}$ & $9.98 \times 10^{-6}$ & $8.87 \times 10^{-7}$ & $9.94 \times 10^{-8}$ \\
& $L_{\infty}$ & $1.65 \times 10^{-2}$ & $7.24 \times 10^{-4}$ & $4.84 \times 10^{-5}$ & $4.34 \times 10^{-6}$ & $4.88 \times 10^{-7}$ \\
& $E_{\text {shp }}$ & $1.91 \times 10^{-1}$ & $7.58 \times 10^{-3}$ & $4.91 \times 10^{-4}$ & $4.36 \times 10^{-5}$ & $4.89 \times 10^{-6}$ \\
$U=10^{5}$, & $L_{1}$ & $1.77 \times 10^{-3}$ & $7.15 \times 10^{-7}$ & $5.14 \times 10^{-10}$ & $3.35 \times 10^{-11}$ & $4.90 \times 10^{-11}$ \\
$V=10^{5}$ & $L_{2}$ & $3.94 \times 10^{-5}$ & $1.61 \times 10^{-8}$ & $1.11 \times 10^{-11}$ & $4.00 \times 10^{-13}$ & $5.82 \times 10^{-13}$ \\
& $L_{\infty}$ & $1.67 \times 10^{-4}$ & $7.55 \times 10^{-8}$ & $5.38 \times 10^{-11}$ & $1.10 \times 10^{-12}$ & $1.56 \times 10^{-12}$ \\
& $E_{\text {shp }}$ & $1.93 \times 10^{-3}$ & $7.91 \times 10^{-7}$ & $5.48 \times 10^{-10}$ & $1.22 \times 10^{-11}$ & $1.71 \times 10^{-11}$ \\
\hline
\end{tabular}

Table 3. Computed $L_{2}$ error norms for Eq. (8) using the Legendre-polynomial finite-element model at different flow directions

\begin{tabular}{llcclll}
\hline$U$ and $V$ & Norm & \multicolumn{1}{c}{$11 \times 11$} & \multicolumn{1}{c}{$21 \times 21$} & \multicolumn{1}{c}{$31 \times 31$} & \multicolumn{1}{c}{$41 \times 41$} & $51 \times 51$ \\
\hline$U=100$, & $L_{1}$ & $8.29 \times 10^{-5}$ & $1.51 \times 10^{-6}$ & $8.46 \times 10^{-9}$ & $6.17 \times 10^{-9}$ & $5.07 \times 10^{-9}$ \\
$V=100$ & $L_{2}$ & $4.41 \times 10^{-6}$ & $7.62 \times 10^{-8}$ & $3.70 \times 10^{-10}$ & $2.87 \times 10^{-10}$ & $2.67 \times 10^{-10}$ \\
& $L_{\infty}$ & $4.54 \times 10^{-5}$ & $7.70 \times 10^{-7}$ & $2.06 \times 10^{-9}$ & $2.06 \times 10^{-9}$ & $2.06 \times 10^{-9}$ \\
& $E_{\text {shp }}$ & $1.66 \times 10^{-4}$ & $2.14 \times 10^{-6}$ & $1.47 \times 10^{-8}$ & $9.77 \times 10^{-9}$ & $7.54 \times 10^{-9}$ \\
$U=100$, & $L_{1}$ & $5.44 \times 10^{-7}$ & $3.71 \times 10^{-8}$ & $1.77 \times 10^{-8}$ & $3.40 \times 10^{-8}$ & $1.86 \times 10^{-8}$ \\
$V=50$ & $L_{2}$ & $1.36 \times 10^{-8}$ & $8.59 \times 10^{-10}$ & $5.54 \times 10^{-10}$ & $7.63 \times 10^{-10}$ & $5.67 \times 10^{-10}$ \\
& $L_{\infty}$ & $8.13 \times 10^{-8}$ & $4.10 \times 10^{-9}$ & $4.10 \times 10^{-9}$ & $4.10 \times 10^{-9}$ & $4.10 \times 10^{-9}$ \\
& $E_{\text {shp }}$ & $1.08 \times 10^{-6}$ & $3.96 \times 10^{-8}$ & $2.00 \times 10^{-8}$ & $2.51 \times 10^{-8}$ & $1.86 \times 10^{-8}$ \\
\hline
\end{tabular}

Table 4. Computed $L_{2}$ error norms for Eq. (8) using the finite-element model of Rice and Schnipke at different flow directions

\begin{tabular}{lllcccc}
\hline$U$ and $V$ & Norm & \multicolumn{1}{c}{$11 \times 11$} & $21 \times 21$ & $31 \times 31$ & $41 \times 41$ & $51 \times 51$ \\
\hline$U=100$, & $L_{1}$ & 1.72 & $4.97 \times 10^{-1}$ & $2.17 \times 10^{-1}$ & $1.17 \times 10^{-1}$ & $7.16 \times 10^{-2}$ \\
$V=100$ & $L_{2}$ & $3.55 \times 10^{-2}$ & $1.09 \times 10^{-2}$ & $4.84 \times 10^{-3}$ & $2.62 \times 10^{-3}$ & $1.62 \times 10^{-3}$ \\
& $L_{\infty}$ & $1.49 \times 10^{-1}$ & $5.10 \times 10^{-2}$ & $2.34 \times 10^{-2}$ & $1.29 \times 10^{-2}$ & $8.00 \times 10^{-3}$ \\
& $E_{\text {shp }}$ & 1.73 & $5.36 \times 10^{-1}$ & $2.38 \times 10^{-1}$ & $1.29 \times 10^{-1}$ & $7.93 \times 10^{-2}$ \\
$U=100$, & $L_{1}$ & 2.29 & $8.81 \times 10^{-1}$ & $4.90 \times 10^{-1}$ & $3.24 \times 10^{-1}$ & $2.37 \times 10^{-1}$ \\
$V=50$ & $L_{2}$ & $4.74 \times 10^{-2}$ & $2.04 \times 10^{-2}$ & $1.20 \times 10^{-2}$ & $8.28 \times 10^{-3}$ & $6.22 \times 10^{-3}$ \\
& $L_{\infty}$ & $2.12 \times 10^{-1}$ & $9.59 \times 10^{-2}$ & $5.62 \times 10^{-2}$ & $3.80 \times 10^{-2}$ & $2.81 \times 10^{-2}$ \\
& $E_{\text {shp }}$ & 1.91 & $6.41 \times 10^{-1}$ & $3.10 \times 10^{-1}$ & $1.83 \times 10^{-1}$ & $1.22 \times 10^{-1}$ \\
\hline
\end{tabular}


render a composite scheme:

$$
A=\left.(1-\alpha) A\right|_{\text {Le gendre polynomial }}+\left.\alpha A\right|_{\text {Rice and Schnipke }}
$$

Establishing the rule to determine $\alpha$ is considered essential to obtaining high-resolution solutions to Eq. (1). The main consideration behind finding a proper choice of $\alpha$ concerns the solution accuracy and the scheme monotonicity.

The cases under consideration are (1) $U=V=250$ with grid number $11 \times 11$, (2) $U=V=500$ with grid number $21 \times 21$, and (3) $U=V=1,000$ with grid number $41 \times 41$. All cases studied here are with the same Peclet numbers, $\mathrm{Pe}_{x}=\mathrm{Pe}_{y}=25$. We plot the $L_{2}$ error norms against $\alpha$ in Figure 3. Common to each curve is the presence of an inflection point, at which the error norm takes on its minimum value. As Figure 3 reveals, errors decrease from two ends of the weighting factor $\alpha$ until the turning point is reached. Decreasing the value of $\alpha$ suggests increasing use of the conditionally monotonic scheme. It has to be remarked that curves having the same value of $\left(\mathrm{Pe}_{x}, \mathrm{Pe}_{y}\right)$ inflect at the same value of $\alpha$. This enlightens that the optimal value of $\alpha$ depends highly on the Peclet number. For the sake of completeness, we have also investigated three other cases: $(U, V)=(250,125)$ with grid number $11 \times 11,(U, V)=(500,250)$ with grid number $21 \times 21$, and $(U, V)=(1,000,500)$ with grid number $41 \times 41$. Under these circumstances, three test conditions are all with the same Peclet number $\left(\mathrm{Pe}_{x}\right.$,

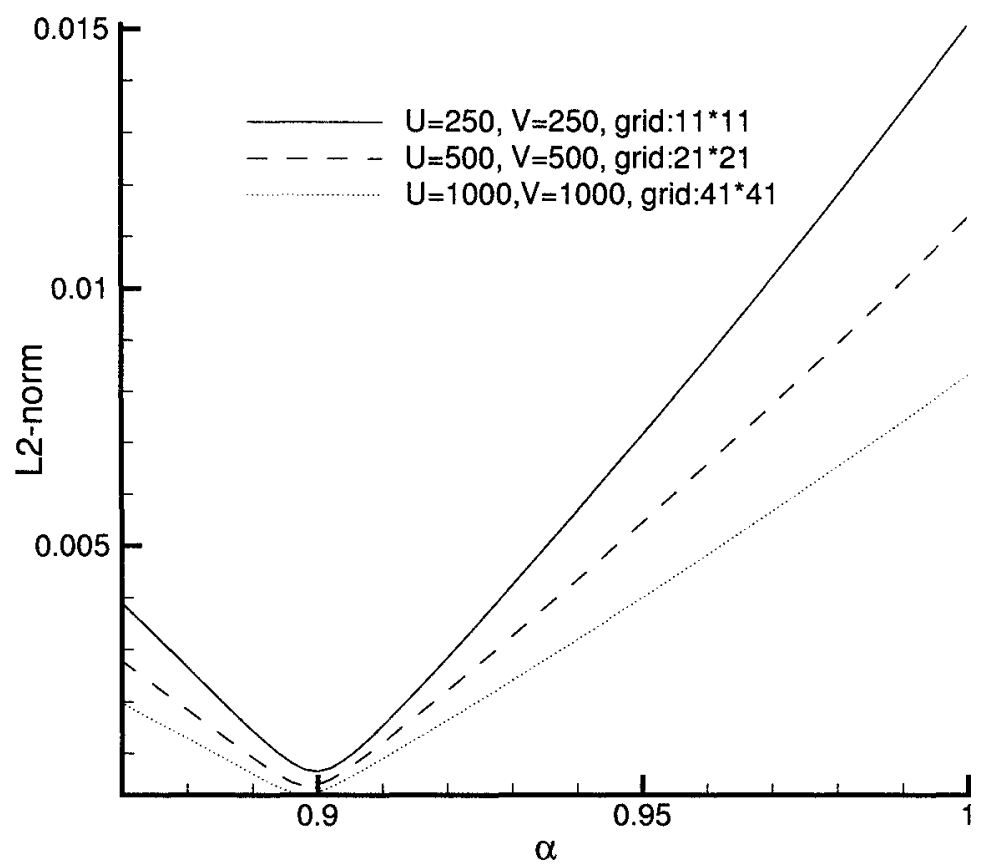

Figure 3. $L_{2}$ error norm distributions against $\alpha$, as shown in (10), for three different conditions which have the same Peclet numbers $\mathrm{Pe}_{x}=\mathrm{Pe}_{y}=25$. 
$\left.\mathrm{Pe}_{y}\right)=(25,12.5)$. We plot the computed error norms against $\alpha$ in Figure 4. As these curves show, inflection points still occur at the same value of $\alpha$.

The above success in applying the composite model to solve problems with different grid sizes and flow angles has important implications on showing that the approach has been extended to a wider Peclet number range. We now turn our attention to constructing a contour map of $\alpha$ so that errors computed from Eq. (10) are minimized. For each pair of Peclet numbers, we plotted the optimal value of $\alpha$ in a map with $\mathrm{Pe}_{x}$ as the abscissa and $\mathrm{Pe}_{y}$ as the ordinate. Given a pair of $\mathrm{Pe}_{x}$ and $\mathrm{Pe}_{y}$, one can find from Figure 5 the weighting factor $\alpha$ which provides the smallest prediction error. It is this contour map of $\alpha$ which gives us a well-established basis for applying this composite scheme to other problems.

Question now arises as to whether or not the choice of $\alpha$, shown graphically in Figure 5, is sensitive to problems being chosen. To answer this question, we consider another model equation which is also amenable to analytic solution. Subject to the boundary condition schematic in Figure 6, the analytic solution for the model scalar transport equation (1) is derived as

$$
\Phi(x, y)=\frac{\left.\sin (\pi y) \exp \left(r_{2} x\right) \exp \left[(V / 2)\left(y-y_{s}\right)\right]\left\{1-\exp \llbracket\left(r_{1}-r_{2}\right)(x-1)\right]\right\}}{\left[1-\exp \left(r_{2}-r_{1}\right)\right] \sin \left(\pi y_{s}\right)}
$$

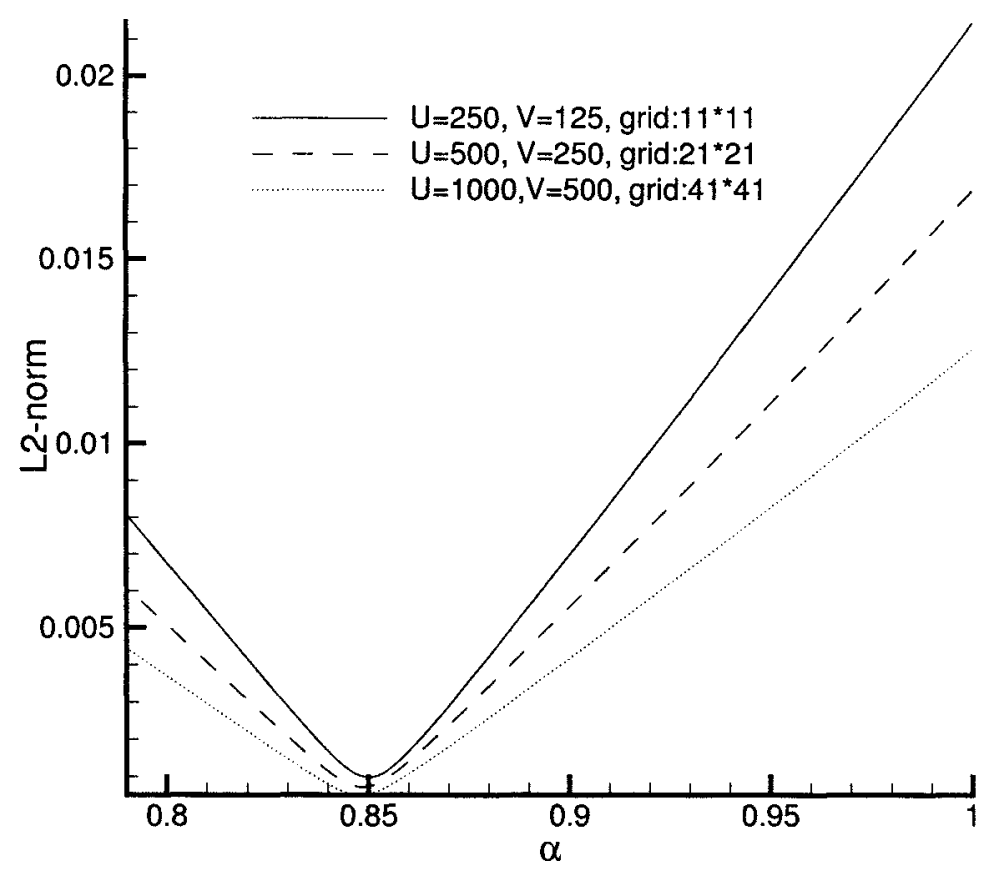

Figure 4. $L_{2}$ error norm distributions against $\alpha$, as shown in (10), for three different conditions which have the same Peclet numbers $\mathrm{Pe}_{x}=25, \mathrm{Pe}_{y}=12.5$. 


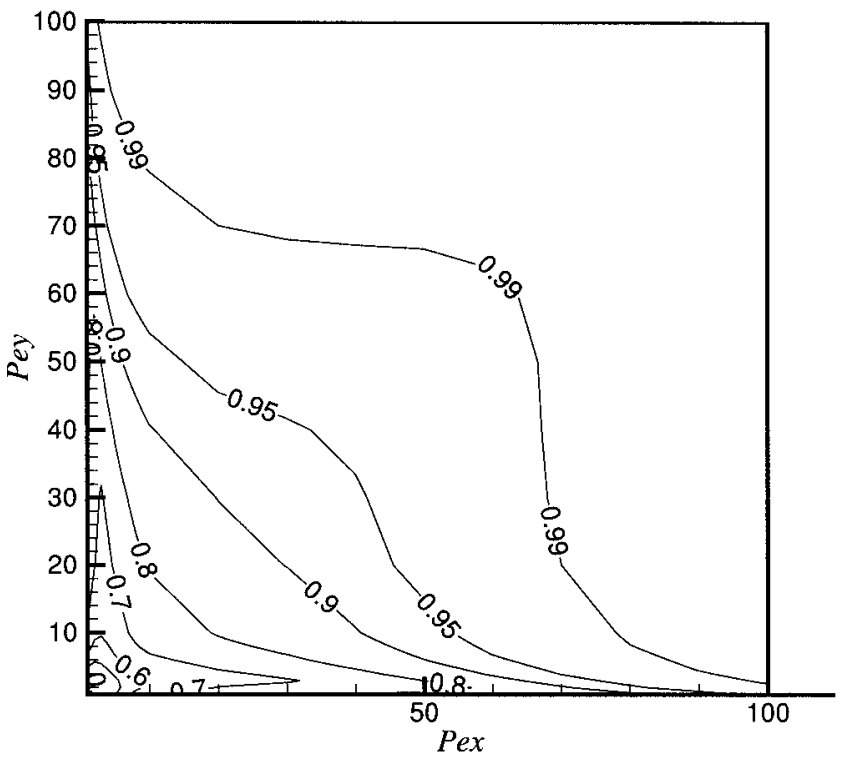

Figure 5. Contour map of the optimal weighting factors $\alpha$.

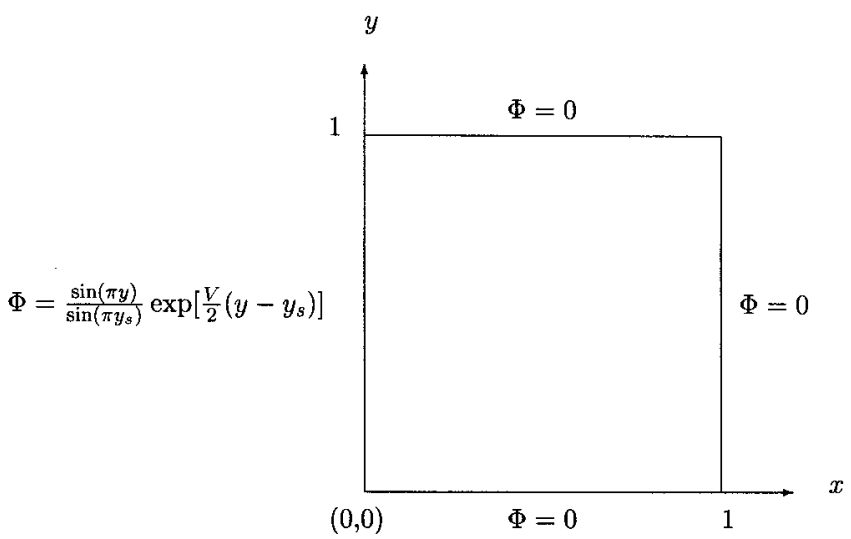

Figure 6. Schematic of the test problem considered for validation. 
where $h=\Delta x=\Delta y$ denotes the grid size and

$$
\begin{aligned}
& U=\frac{1}{4 h} \cot \theta \\
& V=\frac{1}{4 h} \\
& \theta=\tan ^{-1}\left(\frac{V}{U}\right) \\
& y_{s}=1-\frac{1}{\pi} \tan ^{-1}\left(\frac{2 \pi}{V}\right) \\
& r_{1}=\frac{1}{2}\left[U+\sqrt{U^{2}+V^{2}+4 \pi^{2}}\right] \\
& r_{2}=\frac{1}{2}\left[U-\sqrt{U^{2}+V^{2}+4 \pi^{2}}\right]
\end{aligned}
$$

By conducting the time-consuming calculations, we can obtain the optimal $\alpha$ for this problem. What is remarkable from Figure 7 is that the optimal values of $\alpha$ obtained from two different analytic problems essentially follow the same curve.

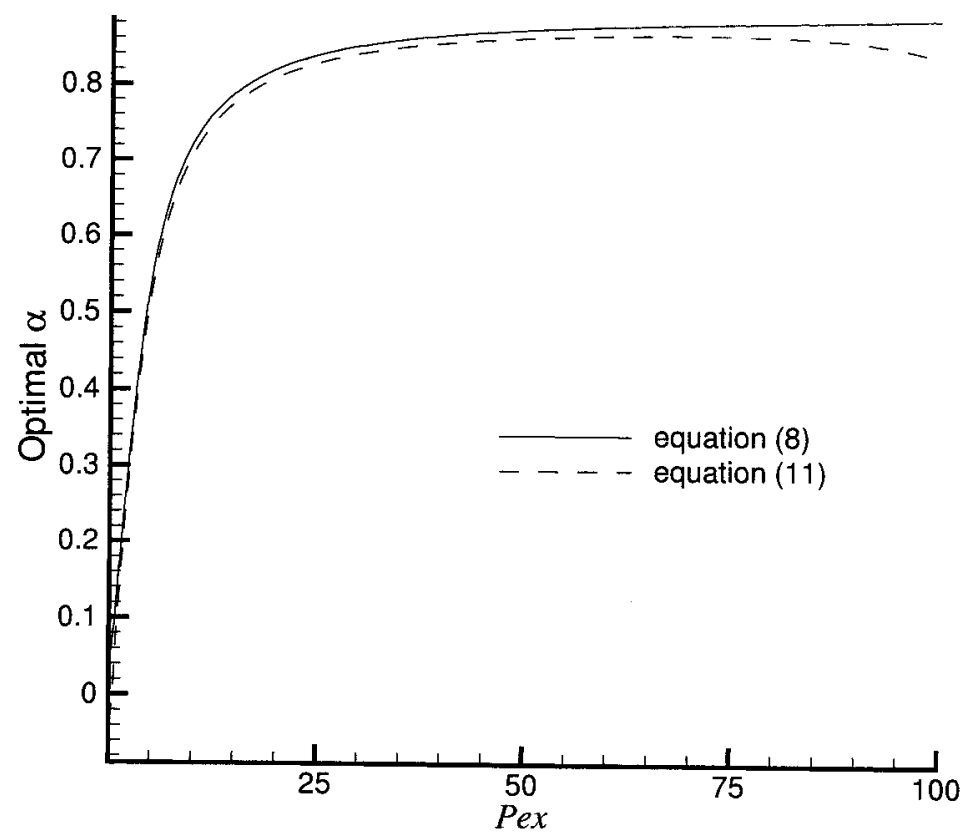

Figure 7. Comparison study of the optimal values of $\alpha$ against Peclet number $\mathrm{Pe}_{x}$ for two test problems. 
This test provides justification for the validity and usefulness of the contour map schematic in Figure 5 at all Peclet numbers.

\section{NUMERICAL RESULTS}

We now turn to studying the skewed transport problem configured in Figure 8. This problem has been chosen to benchmark upwinding schemes to resolve high-gradient solutions in the flow. Inside the square cavity, a tilted line passes through a corner point at $(0,0)$, resulting in a line with a slope of $m=V / U=1$. Across the dividing line, there is a marked change of the solution.

Computational investigations have been performed on uniform grids for the case of $U=100, V=100$. Since this problem is not amenable to analytic solutions, we took the $161 \times 161$ Legendre-polynomial finite-element solutions as the analytic solution for the comparison sake. Figure 9 plotted the composite finiteelement solutions which were computed on $41 \times 41$ grids. For purposes of comparison, we also plotted in Figure 9 other finite-element solutions computed on a much finer grid $(161 \times 161)$. The composite solution compares very favorably with finer solutions; especially noteworthy is the prediction of oscillation-free solution even in the immediate vicinity of the dividing line. This study lends credence to the use of two monotonic schemes in combination to capture high-gradient solutions. For completeness, we also considered the case with $U=10^{5}, V=10^{5}$ and plotted in Figure 10 the computed solution profiles at $y=0.5$. It is evident that the present composite scheme has ability to sharply resolve the solution but at much less computing expense.

Consideration is now given to the case with variable advective velocities. The solution to Eq. (1) is sought in a square $0 \leq x, y \leq 1$, subject to boundary

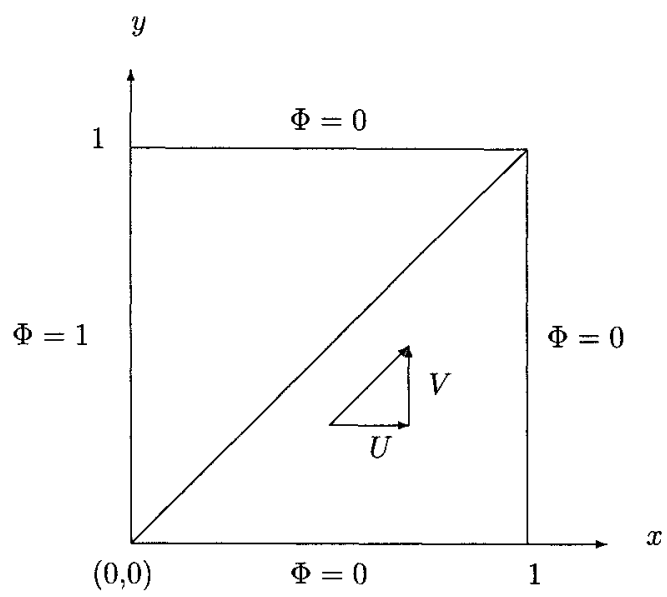

Figure 8. Schematic of the skew advection-diffusion problem. 


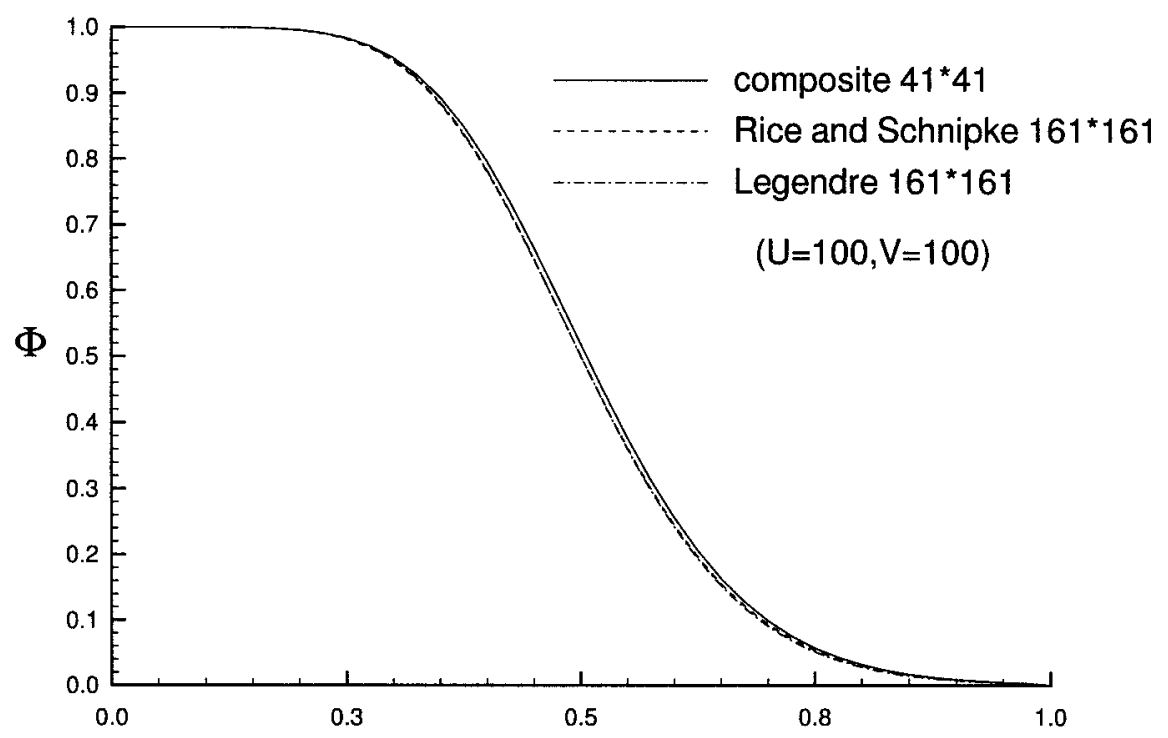

Figure 9. Comparison of computed $\Phi$ at $y=0.5$ for the skew advection-diffusion problem (for the case with $U=V=100$ ).

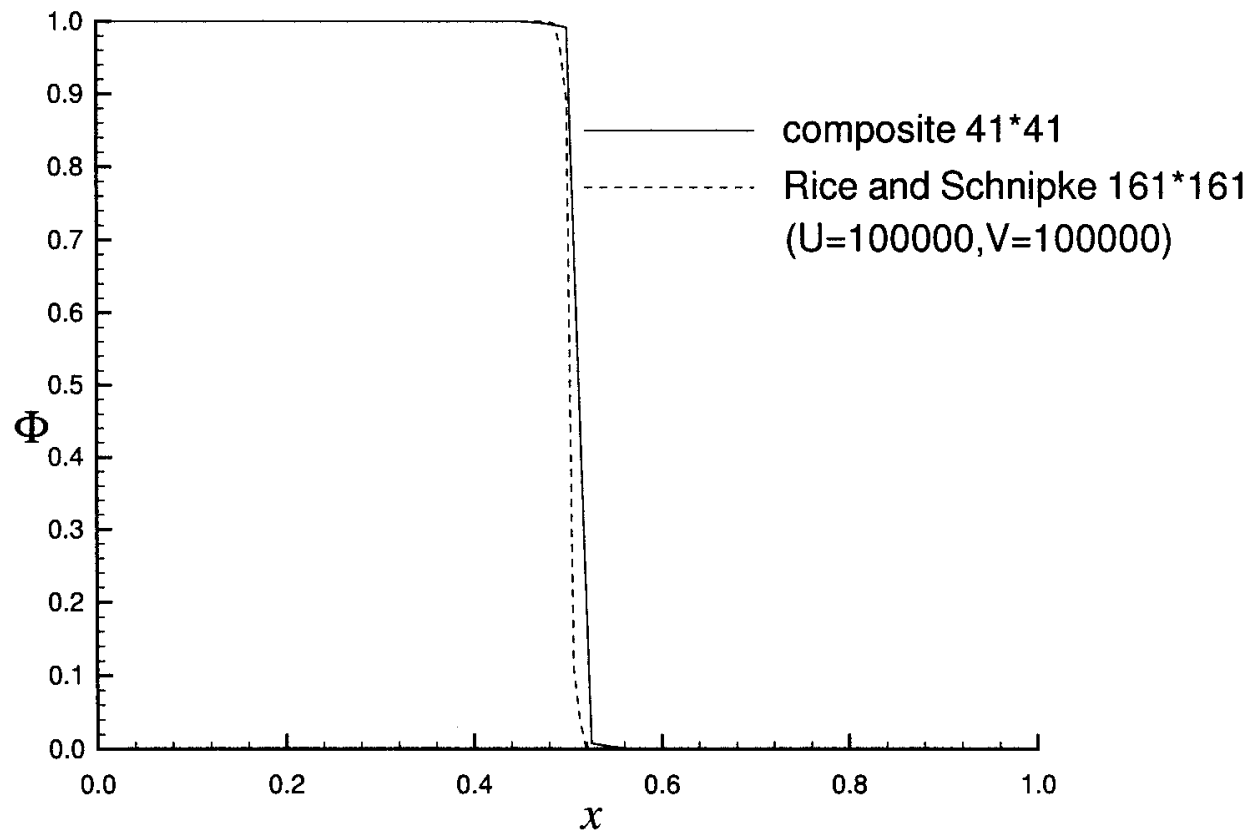

Figure 10. Comparison of sharp profiles of $\Phi$ at $y=0.5$ for the skew advection-diffusion problem (for the case with $U=V=10^{5}$ ). 


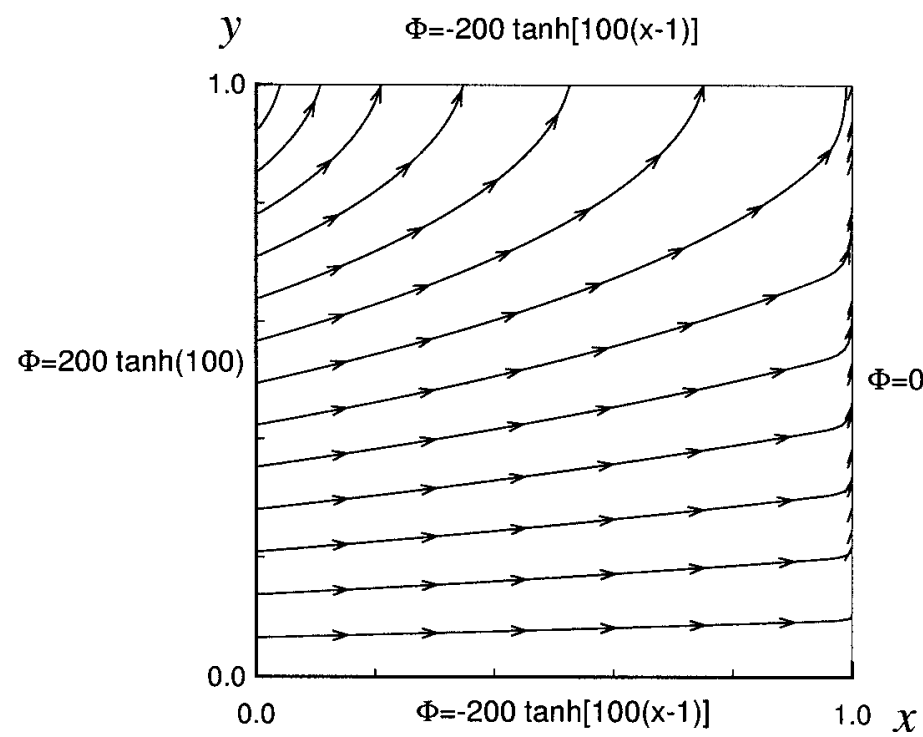

Figure 11. Plots of the streamlines and boundary conditions of the test case with velocity components given in Eq. (13).

conditions given in Figure 11. For the velocity field given below,

$$
\begin{gathered}
U=-2 \lambda \tanh \left[\lambda\left(x-x_{0}\right)\right] \\
V=a_{1} \tan \left(a_{2} y\right)
\end{gathered}
$$

the analytic solution of $\Phi$ takes the form exactly the same as $U$ given in Eq. (13a). The case considered was that of $x_{0}=1, \lambda=100, a_{1}=50$, and $a_{2}=1.5$. Evident in Figure 12 is that the analytic solution of $\Phi$ shows a prevailing boundary-layer character. We present in Figure 12 three computed profiles, which provide justification of the usefulness of the presently proposed composite scheme in both stability and accuracy.

\section{CONCLUDING REMARKS}

We have presented in this article a composite scheme for solving the scalar advection-diffusion transport equation. This article shows the potential advantage that can be gained through the spontaneous use of two monotonic finite-element models. In the construction of finite-element models, we applied the discrete maximum principle as our theoretical basis for judging whether or not solution monotonicity could be obtained. An inherent feature of the first model, featuring the use of Legendre polynomials to span the finite-element space, is its ability to retain the monotonic property as the Peclet numbers fall below 3.6. Use of the scheme of Rice and Schnipke has been shown to considerably improve the monotonicity of the solution since this scheme unconditionally accommodates the 


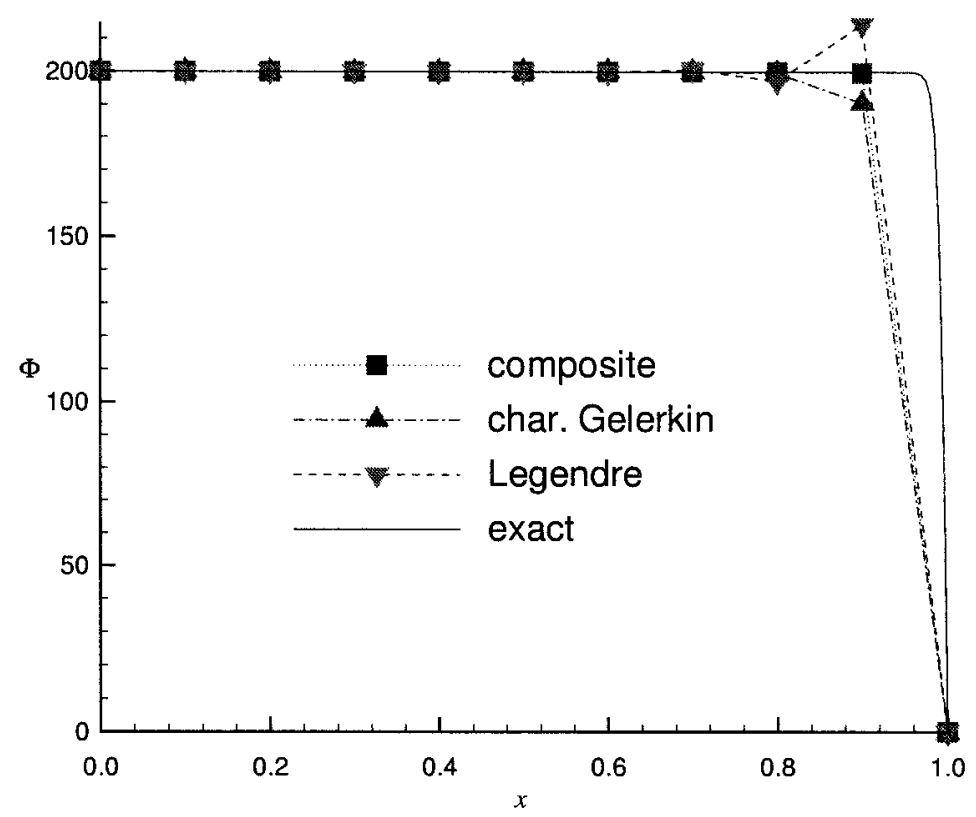

(a)

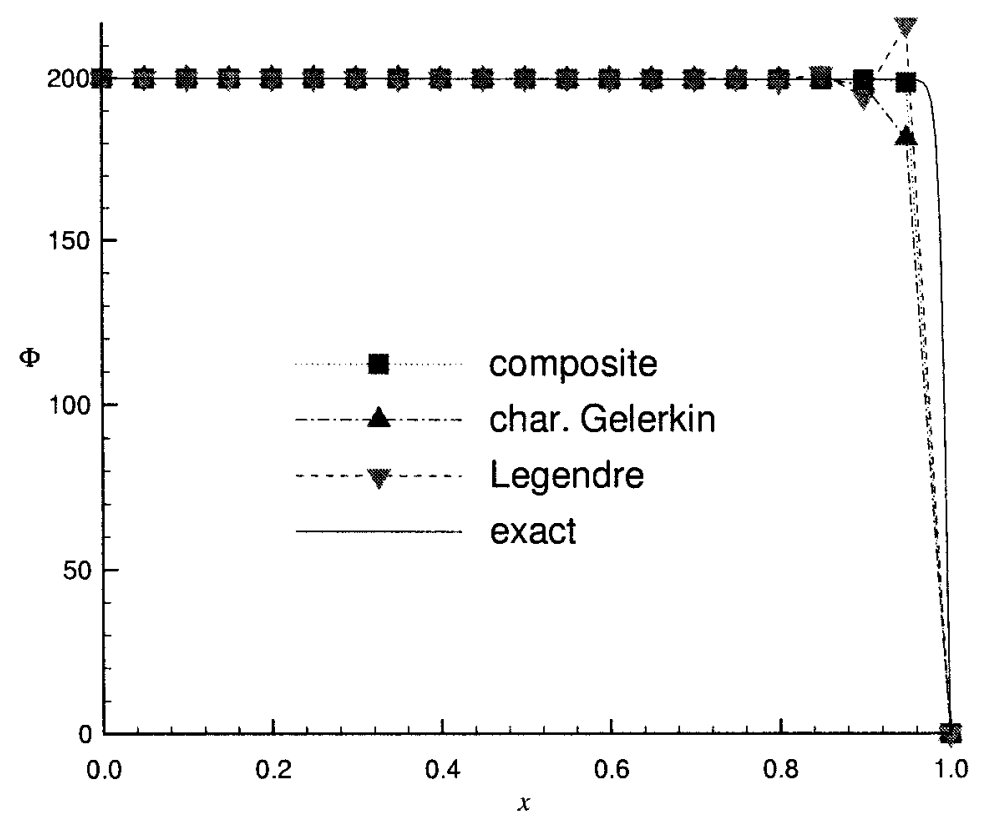

(b)

Figure 12. Computed solutions of $\Phi$ against $x$ at $y=0.5$ using different finite-element models: (a) solutions computed on $11 \times 11$ grids; $(b)$ solutions computed on $21 \times 21$ grids. 
M-matrix property. A potential drawback of this scheme is that it is less accurate in the lower Peclet number range. It is thus tempting to apply two monotonic schemes in combination to complement each other. The key to success of constructing the composite scheme to improve monotonicity and accuracy of the solution for all Peclet numbers is rooted in the theoretical weighting factor derived in this article.

\section{REFERENCES}

1. A. Harten, High Resolution Schemes for Hyperbolic Conservation Law, J. Comput. Phys., vol. 49, pp. 357-393, 1983.

2. S. Spekreijse, Multigrid Solution of Monotone Second-Order Discretizations of Hyperbolic Conservation Laws, Math. Comput., vol 49, pp. 135-155, 1987.

3. J. G. Rice and R. J. Schnipke, A Monotone Streamline Upwind Finite Element Method for Convection-Dominated Flows, Comput. Meth. Appl. Mech. Eng., vol. 47, pp. 313-327, 1983.

4. M. Ahue's and M. Telias, Petrov-Galerkin Scheme of the Steady State Convection Diffusion Equation, Finite Elements in Water Resources, vol. 2/3, 1982.

5. T. W. H. Sheu, M. M. T. Wang, and S. F. Tsai, A Petrov-Galerkin Finite Element Model for Analyzing Incompressible Flows at High Reynolds Numbers, Int. J. Comput. Fluid Dynam., vol. 5, pp. 213-230, 1995.

6. T. W. H. Sheu, S. F. Tsai, and M. M. T. Wang, A Monotone Multidimensional Upwind Finite-Element Method for Advection-Diffusion Problems, Numer. Heat Transfer B, vol. 29, pp. 325-344, 1996.

7. T. W. H. Sheu, S. F. Tsai, and M. M. T. Wang, A Monotone Finite Element Method with Test Space of Legendre Polynomials, Comput. Meth. Appl. Mech. Eng., vol. 143, pp. 349-372, 1997.

8. T. W. H. Sheu, S. F. Tsai, and S. K. Wang, Monotonic, Multi-dimensional Flux Discretization Scheme for All Peclet Numbers, Numer. Heat Transfer B, vol. 31, no. 4, pp. 441-457, 1997.

9. T. Meis and U. Marcowitz, Numerical Solution of Partial Differential Equations. In Applied Mathematical Science, vol. 32, Springer-Verlag, New York, 1981.

10. T. Ikeda, Maximal Principle in Finite Element Models for Convection-Diffusion Phenomena, in Numerical and Applied Analysis, vol. 4, North-Holland, Kinokuniya, Amsterdam, Tokyo, 1983.

11. Tony W. H. Sheu, S. F. Tsai, and M. M. T. Wang, Discussion of Numerical Deficiency of Applying a Partially Weighted Upwind Finite-Element Model to Incompressible Navier-Stokes Equations, Numer. Heat Transfer B, vol. 32, no. 2, pp. 197-214, 1997. 